\title{
Calidad de vida en pacientes con implante coclear en Hospital Barros Luco Trudeau
}

\section{Quality of life in patients with cochlear implants in Barros Luco Trudeau Hospital}

\author{
Carlos Morales A', Karimme Morales A², Maritza Rahal E
}

\begin{abstract}
RESUMEN
Introducción: El concepto de calidad de vida se ha tornado de suma importancia y actualmente toda intervención sanitaria es valorada por el impacto que causa en esta misma. La hipoacusia bilateral profunda produce importantes consecuencias, retrasando la adquisición del lenguaje, disminuye significativamente el aprendizaje y produce dificultades en cómo se desarrolla en su entorno. El implante coclear es el tratamiento de elección en esta patología y es de suma importancia conocer el impacto real que tiene en la calidad de vida de aquellos sometidos a esta intervención.

Objetivo: Conocer la calidad de vida global de los pacientes implantados en el Hospital Barros Luco Trudeau entre 2015 y 2016, usando el instrumento de medición de calidad de vida KINDL.

Material y método: Estudio analítico prospectivo de cohorte, para medición de calidad de vida se utilizó el instrumento KINDL, se aplicó instrumento a menores implantados y padres a través de entrevista directa en periodos preimplante, a 6 meses y al año de implantación, datos y pruebas estadísticas se realizaron con software SPSS 13.

Resultados: Se estudiaron 16 pacientes en edad pediátrica implantados en Hospital Barros Luco Trudeau. Se evidenciaron cambios en la calidad de vida global y en las subescalas de bienestar emocional, autoestima, educación, relación con amigos y familia, además de percepción de enfermedad.

Conclusiones: El implante coclear en nuestra serie demostró una modificación positiva en la calidad de vida global tanto de los pacientes como de su entorno familiar.

Palabras clave: Implante coclear, hipoacusia, calidad de vida, KINDL.
\end{abstract}

\begin{abstract}
Introduction: Quality of life has become very important, and nowadays all health interventions are valued for the impact they can have on it. Profound bilateral hearing loss produces major consequences such as delaying the acquisition of language, which results in a significant decrease in learning and creates difficulties in how the individual develops in their environment. The cochlear implant is the treatment of choice in this pathology, and it is imperative to know the real impact it has on the quality of life of those subjected to this intervention.
\end{abstract}

\footnotetext{
1 Médico, Servicio Otorrinolaringología Hospital Barros Luco Trudeau, Santiago, Chile.

2 Socióloga, Universidad de Valparaíso, Chile.

Recibido el 19 de noviembre, 2017. Aceptado el 11 de marzo, 2018.
} 
Aim: To know the overall quality of life of the patients implanted in the Barros Luco Trudeau Hospital between 2015 and 2016, using the KINDL quality of life measurement instrument.

Material and method: Prospective analytical cohort study, to measure the quality of life, the KINDL instrument was used. The instrument was applied to implanted minors and their parents through a direct interview in pre-implantation periods at six months and after one year of implantation. The data and statistics tests were made with SPSS 13 software.

Results: 16 pediatric patients implanted in the Barros Luco Trudeau Hospital were studied. There were changes in the global quality of life and the subscales of emotional well-being, such as the patient's self-esteem, education, relationships with friends and family, as well as the perception of illness.

Conclusions: The cochlear implant in our series demonstrated a positive change in the global quality of life for both patients and their family environments.

Key words: Cochlear implant, hearing loss, life quality, KINDL.

\section{INTRODUCCIÓN}

La medición del impacto que las intervenciones médicas tienen sobre la calidad de vida de nuestros pacientes está adquiriendo una importancia cada vez mayor en el ejercicio de la medicina moderna, y en muchas ocasiones prima sobre los resultados concretamente médicos ${ }^{1}$. La forma tradicional de medir la efectividad de un procedimiento 0 intervención, basada en el estudio de la mortalidad y la morbilidad, se muestra poco útil cuando analizamos tratamientos que no buscan como objetivo mantener la vida de un paciente pero que sí tratan de alcanzar una mejoría de esta misma ${ }^{2,3}$.

La hipoacusia profunda bilateral genera importantes consecuencias. A nivel individual afecta la adquisición de lenguaje, retrasa el aprendizaje, disminuye las habilidades cognitivas y sociales, se asocia a problemas de comportamiento en los distintos ambientes de interacción, menor bienestar psicológico y disminuye la productividad laboral ${ }^{4}$. Asimismo, tanto los padres, familiares 0 cuidadores de niños hipoacúsicos presentan un importante estrés físico-psicológico ${ }^{5}$. Por lo tanto, esta patología tiene una importante carga de enfermedad asociada, no solo al paciente, sino que también en su entorno.

Desde un punto de vista económico, el implante coclear corresponde a una intervención costosa, pero cuyos resultados sobrepasan con creces la inversión realizada ${ }^{6}$, disminuyendo tanto los gastos personales, como los años de vida saludables perdidos relacionados con la hipoacusia. Con el implante coclear se logra un desarrollo de lenguaje oral en el paciente, facilitando la asistencia a una institución educativa regular. En consecuencia, los costos en educación son menores comparados con los que requieren los individuos con otro tipo de ayudas auditivas ${ }^{7}$. Además de generar un ahorro económico, este dispositivo mejora el estado de salud del paciente, compensando así el valor de la intervención quirúrgica que traduce una intervención como éstå .

Está ampliamente reconocido que el implante coclear es el único tratamiento eficaz para las hipoacusias profundas y aquellas severas en las que no resultan de utilidad los audífonos. Numerosos trabajos demuestran sus beneficios en cuanto a la mejoría de la audición de los pacientes implantados ${ }^{9}$. Sin embargo, el impacto que estos dispositivos tienen sobre la vida diaria de los pacientes está aún poco explorado, existiendo algunas publicaciones como la de Castro $^{10}$ en España, donde objetiva una mejoría tanto en resultados auditivos como en la calidad de vida de los pacientes tratados. Sin embargo, en nuestro país no existen publicaciones al respecto.

No podemos olvidar que la OMS define la salud como el completo estado de bienestar físico, mental y social. De esta forma, los métodos de medición de bienestar en salud como los cuestionarios de calidad de vida nos proporcionan la herramienta más adecuada para la cuantificación del nivel de salud de un individuo.

Es por esto, y dado los recursos tanto humanos como materiales gestionados en esta intervención, que se hace necesario aproximarnos a conocer el impacto que tiene el tratamiento, en la 
calidad de vida de nuestros pacientes, más allá del ya conocido beneficio meramente auditivo.

Debido a esto nos planteamos como hipótesis de investigación si el implante coclear es un modificador positivo de la calidad de vida, es por ello que nuestro objetivo general fue el de conocer la calidad de vida global de estos pacientes a través de un instrumento de medición de ésta previamente validado.

\section{MATERIAL Y MÉTODO}

\section{Pacientes}

El Ministerio de Salud de Chile en el año 2003 implementó un programa de implantes cocleares para pacientes con hipoacusia profunda bilateral, siendo uno de los centros de referencia el Hospital Barros Luco Trudeau. Actualmente, ya se han realizado más de 170 implantes cocleares entre población adulta y pediátrica, siendo nuestros pacientes no solo de la capital de nuestro país, Santiago, sino que muchos de ellos derivados de todo Chile.

Para este estudio se reclutaron pacientes pediátricos implantados desde el año 2015 a 2016, con el propósito de realizar una cohorte con un seguimiento a 6 meses y 1 año poscirugía. Con el objetivo de realizar una medición de la calidad de vida se utilizó el cuestionario KINDL adaptado, con una estructura y consistencia interna demostrada $^{11}$ y validado al español ${ }^{12}$, que comprende distintas formas para niños separado por grupos etarios entre 3 a 7 años; 8 a 17 años y otra forma para padres. Este formato del instrumento permite evaluar por separado la percepción de calidad de vida del niño y de los padres, lo cual nos hizo elegir esta encuesta por sobre otras registradas en la literatura.
A través de consentimiento informado a los padres se realizó el ingreso al estudio, siguiendo los criterios de inclusión: Pacientes pediátricos entre los 3 y 17 años, portadores de hipoacusia profunda bilateral o severa con indicación de implante coclear, intervenidos en nuestro centro durante el periodo estudiado, y que comprendan el cuestionario por sí solos o con asistencia de sus padres. Se excluyeron del estudio los pacientes que no dieron su consentimiento o no lograron comprender el cuestionario aplicado.

El instrumento se aplicó en 3 etapas, tanto a los pacientes como a sus padres en las distintas formas que comprende el instrumento: 1) Previo a la cirugía, 2) A los 6 meses posterior a la cirugía y 3) Después de 1 año del implante coclear. El método utilizado fue a través de entrevista directa, y en pacientes fuera de Santiago se utilizó la encuesta telefónica. Este estudio fue aprobado por el Comité de Ética del Servicio de Salud Metropolitano Sur.

\section{Cuantificación de la calidad de vida}

Para realizar la medición de la calidad de vida fue utilizado como referencia el cuestionario KINDL, el cual consiste en 24 preguntas igualmente distribuidas en 6 subescalas: bienestar físico, bienestar psicológico, autoestima, familia, amigos, desempeño educacional. Esto se acompaña de un apartado de "enfermedad" en el caso que exista estadía hospitalaria, como es el caso de esta patología. Cada subescala posee un puntaje que en combinación con cada uno de ellos resulta en un escore total que intenta reflejar la calidad de vida de 0 a 100 puntos. Este formato se desarrolla a través de consultas que son resueltas con las siguientes respuestas: Nunca, casi nunca, algunas veces, casi siempre y siempre. En la Tabla 1 se pueden visualizar ejemplo de las consultas incluidas en la encuesta.

Tabla 1.

\begin{tabular}{|ll|}
\hline Subescala & Pregunta \\
\hline Bienestar físico & ¿Durante la semana pasada te has sentido fuerte o con energía? \\
Bienestar emocional & ¿Durante la semana pasada te has divertido o reído? \\
Autoestima & ¿Durante la semana pasada has tenido buenos pensamientos? \\
Familia & ¿Durante la semana pasada te has llevado bien con tus padres? \\
Amigos & ¿Durante la semana pasada te has sentido diferente a tus amigos? \\
Educación & ¿Durante la semana pasada has sentido que pudiste realizar bien tus tareas en el colegio? \\
Enfermedad & ¿Durante la semana pasada te has sentido enfermo o decaído? \\
\hline
\end{tabular}




\section{Análisis estadístico}

El análisis se llevó a cabo en 3 secciones dadas por las formas de la encuesta: Niños menores entre 3 y 7 años, pacientes entre 8 y 17 años y padres. Se caracterizaron las muestras, y se realizó el análisis describiendo variables continuas con medias, promedios y desviación estándar. Las variables cualitativas se describen con frecuencias y porcentajes. El score por subescala y su comparación se realiza a través de prueba estadística chi-cuadrado.

\section{RESULTADOS}

Se obtuvo una muestra de 16 pacientes, que junto a sus padres, corresponde a una aplicación de 92 encuestas en total en las 3 etapas de medición, no completando el total, dado a dificultades en la comprensión del instrumento en pacientes menores. El $100 \%$ de los pacientes presentaba el diagnóstico de hipoacusia sensorioneural profunda bilateral, el $58 \%$ de ellos correspondía a etapa prelingual. La muestra integrada por los menores de 7 años, la distribución por sexo es de forma igualitaria, donde sus edades van de los 3 a los 6 años, siendo los 3 años la edad absoluta más recurrente. Todos los pacientes presentaban más de 1 hermano dentro de su grupo familiar, lo cual implica mayores dificultades en el proceso de rehabilitación dado el tiempo invertido en el cuidado de éstos (Figura 1).

Realizando el análisis por subescalas y tomando como ejemplo algunas de las consultas:

Bienestar físico: Antes de la operación 66,6\% declara haberse sentido Muchas veces o Alguna vez ante la afirmación "Durante la semana pasada me he sentido enfermo". A los seis meses de posoperatorio el $50 \%$ declara que Alguna vez se sintió enfermo durante la semana pasada y $50 \%$ afirma que Nunca se sintió enfermo durante la semana pasada. Después de un 1 año de postoperatorio $66,7 \%$ afirma haberse sentido Alguna vez enfermo la semana pasada y $33,3 \%$ afirmó que Nunca se sintió enfermo la semana pasada. Diferencias no significativas en este ámbito.
Bienestar emocional: En cuanto al estado anímico de los niños y niñas, previo a la operación $50 \%$ afirma que durante la semana pasada Alguna vez se ha reído y divertido mucho; y el otro $50 \%$ afirma que durante la semana pasada Muchas veces se ha reído o divertido. Posteriormente, en el segundo y tercer levantamiento de datos, se mantienen las cifras, donde $33,3 \%$ afirma que Alguna vez se ha divertido mucho y $66,7 \%$ afirma que Muchas veces durante la semana pasada se ha reído o divertido mucho.

Autoestima: La afirmación en cuanto al orgullo propio y la subescala en total, da cuenta que previo a la operación el $100 \%$ de los niños y niñas afirma que durante la semana pasada se sintió Muchas veces orgulloso u orgullosa de mi mismo/a, luego a pesar de que después de 6 meses este porcentaje baja 33,3\%, al año vuelve en 100\% de afirmación.

Familia: Las relaciones parentales, como dimensión fundamental en la calidad de vida de los niños y niñas, muestra que previo a la operación 83,3\% afirmó que durante la semana pasada se llevó bien

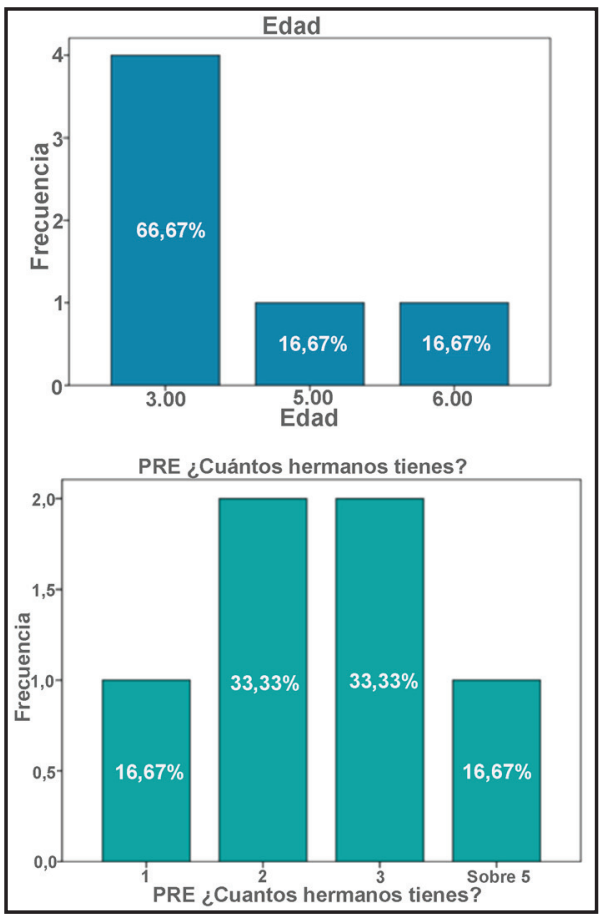

Figura 1. 
Muchas veces con sus padres, porcentaje que va aumentando, luego de un año de posoperatorio el $100 \%$ de los niños y niñas lo afirma. Demostrando un cambio importante en esta subescala.

Amigos: En cuanto a las relaciones con sus pares, previo a la operación el 100\% afirmó haber jugado Muchas veces con sus amigos durante la semana pasada y $66,3 \%$ además afirmó que Muchas veces se llevó bien con ellos. En el tercer momento de encuesta, a un año de la operación, el 100\% de los niños y niñas afirma que ha jugado con sus amigos y que se ha llevado bien con ellos, durante la semana previa a la encuesta.

Educación: En el desarrollo académico, previo a la operación y posoperación se mantiene invariables, el $66,7 \%$ afirma que durante la semana pasada Muchas veces cuando estuvo en el kínder o jardín, pudo hacer sus tareas. En el ambiente escolar, previo a la operación el $66,7 \%$ afirmó que durante la semana pasada lo ha pasado bien, cifra que se mantiene después de un año de la operación.

Enfermedad: La enfermedad ha sido parte importante en la historia vital (el $100 \%$ de los niños afirma haber estado enfermo por mucho tiempo), es por tanto que como experiencia de vida, ésta podría tener una incidencia en las expectativas y autoconcepto. En cuanto al sentimiento de tristeza 0 pena que genera la enfermedad, ante la afirmación "Durante la semana pasada me he encontrado triste por mi enfermedad", previo a la operación el $100 \%$ de los niños afirma que Muchas veces o Alguna vez, durante la semana pasada se encontró triste por la enfermedad. Pasado 6 meses luego de la operación el 50\% afirmó que Alguna vez se sintió triste y el otro 50\% afirmó que Nunca se encontró. En el tercer levantamiento estos datos no variaron mostrando un cambio significativo.

Ante la afirmación: "Durante la semana pasada no he querido que nadie se diera cuenta de mi enfermedad" previo a la operación el 83,3\% de Ios niños y niñas afirma que Muchas veces no ha querido que nadie se diera cuenta, posteriormente a los 6 meses esta cifra disminuye a $66,7 \%$, para luego de un año disminuir al $50 \%$. En cuanto a las relaciones sociales recreacionales en la comunidad escolar, previo a la operación el $66,7 \%$ de los niños y niñas afirma que Muchas veces durante la semana pasada se ha perdido de actividades escolares a causa de la enfermedad, a los 6 meses posteriores de la operación el 83,3\% afirmo que Alguna vez durante la semana pasada se ha perdido de actividades escolares a causa de su enfermedad. Pasado un año, en el tercer levantamiento de datos, el $66,7 \%$ afirmó que Alguna vez se ha perdido actividades y $33,3 \%$ afirmó que Nunca se perdió de éstas a causa de la enfermedad. El resumen del score en este grupo se puede visualizar en la Tabla 2.

En el grupo entre 7 y 17 años al observar la caracterización de la muestra se puede apreciar que $60 \%$ son niños y $40 \%$ niñas, $30 \%$ de los consultados/as declara tener 6 años, y respecto al régimen administrativo de su institución educacional un $90 \%$ acude a escuelas públicas (Figura 2).

Realizando el análisis por subescalas, destacando las principales consultas:

Bienestar físico: Al consultar a los/as adolescentes si les ha dolido algo, ellos consideran que: previo a la operación $50 \%$ le ha sucedido Algunas veces, mientras que a $30 \%$ le ha dolido Casi nunca y a 20\% Nunca, 6 meses posterior a la operación se

Tabla 2

\begin{tabular}{|lllllllll|}
\hline & $\begin{array}{l}\text { Bienestar } \\
\text { fisico }\end{array}$ & $\begin{array}{l}\text { Bienestar } \\
\text { emocional }\end{array}$ & Autoestima & Familia & Amigos/as & Escuela & Enfermedad & Suma \\
\hline Preoperación & 21 & 12,5 & 16 & 17 & 16,5 & 16,5 & 33,5 & 77 \\
Posoperación 6 meses & 15 & 15 & 16,5 & 16,5 & 17 & 15,5 & 12,83 & 64,48 \\
Posoperación 1 año & 14 & $16^{*}$ & 17,5 & 18,5 & $21^{*}$ & 18,5 & $44,5^{*}$ & $83,6^{*}$ \\
\hline
\end{tabular}

*Significancia estadística $p<0,05$. 
ha hecho la misma pregunta y $40 \%$ considera que Casi nunca le ha dolido, pese a ello $20 \%$ de los aludidos declara que le ha dolido Algunas veces - Casi siempre. Al consultarles un año posterior a la operación $50 \%$ considera que Casi nunca le ha dolido, 20\% Casi siempre o Algunas veces y $10 \%$ de los consultados Nunca ha sentido dolor tras el año de operación. Llama la atención que tras el seguimiento $20 \%$ declare sentir dolor Casi siempre a los 6 meses y al año, como también que a los 6 meses 20\% declare sentir dolor Siempre, ello se puede deber a los malestares propios de la recuperación tras la intervención (Figura 3).

Bienestar emocional: Destaca que al preguntar a los adolescentes si han sentido miedo o se han sentido inseguros, éstos consideran lo siguiente: previo a la operación $60 \%$ de ellos se ha sentido inseguro Algunas veces, 30\% Casi nunca y 10\% Nunca, transcurridos 6 meses de la operación 30\% se ha sentido inseguro o con miedo Casi siempre 0 Algunas veces y $20 \%$ Casi nunca o Nunca. Después de un año $50 \%$ se ha sentido inseguro o con miedo Algunas veces y $30 \%$ lo ha hecho Casi siempre, $20 \%$ Casi nunca ha sentido miedo o inseguridad.

Autoestima: Al consultar si se han gustado a sí mismos/as han declarado lo siguiente: previo a la operación $40 \%$ se ha gustado Algunas veces 0 Casi siempre. Después de un año de la operación $40 \%$ de los encuestados se ha gustado a sí mismos Algunas veces, 30\% lo hace Casi siempre, y el resto Siempre. Lo que demuestra un cambio estadísticamente significativo.

Familia: Cuando se consulta a los adolescentes diagnosticados con hipoacusia si se han sentido a gusto en casa previo a la operación, $40 \%$ considera que se ha sentido a gusto Casi siempre o Siempre. A los 6 meses después de la intervención se presenta una tendencia al alza la que se mantiene al año después de la operación, donde a la misma pregunta se obtiene que $50 \%$ considera que Siempre se ha sentido a gusto en casa, $40 \%$ lo hace Casi siempre y $10 \%$ Algunas veces. Siendo significativo el cambio.

Amigos: Destaca en esta subescala la diferencia ante la consulta si se han sentido diferente a sus

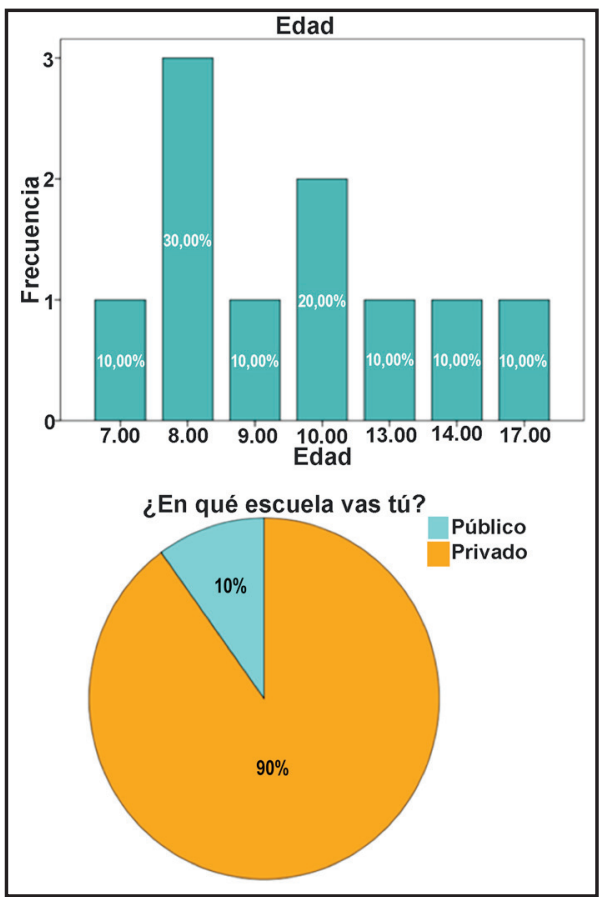

Figura 2.

\begin{tabular}{|llr|}
\hline Me ha dolido algo & Frecuencia & Porcentaje \\
\hline Preoperación & Siempre & 0,0 \\
& Casi siempre & 0,0 \\
& Algunas veces & 50,0 \\
& Casi nunca & 30,0 \\
& Nunca & 20,0 \\
Posoperación 6 meses & Siempre & 0,0 \\
& Casi siempre & 20,0 \\
& Algunas veces & 20,0 \\
& Casi nunca & 40,0 \\
Posoperación 1 año & Nunca & 20,0 \\
& Siempre & 0,0 \\
& Casi siempre & 20,0 \\
& Algunas veces & 20,0 \\
& Casi nunca & 50,0 \\
& Nunca & 10,0 \\
\hline
\end{tabular}

Figura 3.

pares, demostrando una mejoría significativa en el periodo estudiado (Figura 4).

Educación: Después de consultar si le ha preocupado su futuro en el colegio, previo a la operación $40 \%$ considera que ello ha ocurrido Casi nunca, 30\% dice que ha ocurrido Nunca, 


\begin{tabular}{|llr|}
\hline $\begin{array}{l}\text { Me he sentido diferente } \\
\text { a los/as demás }\end{array}$ & Frecuencia & Porcentaje \\
\hline Preoperación & & \\
& Siempre & 0,0 \\
& Casi siempre & 40,0 \\
& Algunas veces & 40,0 \\
& Casi nunca & 0,0 \\
Posoperación 6 meses & Nunca & 20,0 \\
& Siempre & 0,0 \\
& Casi siempre & 10,0 \\
& Algunas veces & 30,0 \\
& Casi nunca & 40,0 \\
Posoperación 1 año & Nunca & 20,0 \\
& Siempre & 0,0 \\
& Casi siempre & 10,0 \\
& Algunas veces & 20,0 \\
& Casi nunca & $40,0^{*}$ \\
& Nunca & $30,0^{*}$ \\
\hline
\end{tabular}

Figura 4.

$20 \%$ Algunas veces y $10 \%$ Casi siempre, al año posterior a la operación $50 \%$ se ha preocupado por su futuro Casi siempre, 30\% Siempre y $20 \%$ Algunas veces. Esto demuestra la generación de nuevas expectativas para los jóvenes respecto a esta temática.

Enfermedad: Destaca que frente a la pregunta si han tenido miedo a que la enfermedad empeore, los consultados han respondido: previo a la operación $60 \%$ Casi siempre, $10 \%$ Siempre o Algunas veces y $20 \%$ Nunca. Un año después de la operación $50 \%$ ha tenido miedo Algunas veces, 30\% Casi nunca y $10 \%$ Casi siempre o Nunca. Se puede ver que previo a la operación el miedo a la enfermedad es constante, posterior a la operación a los 6 meses y al año dicho miedo disminuye considerablemente, producto del avance en el proceso de recuperación.
El resumen de la aplicación del instrumento se puede visualizar en la Tabla 3.

Respecto a la evaluación de la consulta a los padres en los dos grupos estudiados cabe destacar que en $83,3 \%$ de los casos es la madre quien respondió el cuestionario, solo un padre participó del estudio. La valorización de la calidad de vida de sus hijos fue la siguiente:

- Tras el seguimiento realizado, ellos consideran que posterior a la intervención quirúrgica a sus hijos e hijas se han sentido menos enfermos/as, evidencia de ello es que previo a la operación los padres declaran que sus hijos e hijas se han sentido enfermos Siempre o Casi siempre en 100\%. Posteriormente a los 6 meses de la operación y al año de ella la tasa de malestar desciende y al año el $100 \%$ de los consultados declara que sus hijos se sienten enfermos Nunca o Casi nunca. Cambio significativo al aplicar la prueba estadística.

- En relación a la subescala de educación destaca que al consultar a la madres y padres si sus hijos/ as han tenido dificultades con sus profesores 0 cuidadores, previo a la operación $66,7 \%$ las ha tenido Casi siempre y $16,7 \%$ Algunas veces 0 Siempre, mientras que al año después $66,7 \%$ lo ha hecho solo Algunas veces y $16,7 \%$ Casi nunca o Nunca. Al observar el ítem en su conjunto se puede apreciar que previo a la operación existían más conflictos con los profesores y cuidadores, disminuyendo posterior a la operación (Tabla 4).

\section{DISCUSIÓN}

La medición de la calidad de vida es sin duda la real valoración que podemos darle a una intervención

Tabla 3.

\begin{tabular}{|lllllllll|}
\hline & $\begin{array}{l}\text { Bienestar } \\
\text { físico }\end{array}$ & $\begin{array}{l}\text { Bienestar } \\
\text { emocional }\end{array}$ & Autoestima & Padres & Amigos & $\begin{array}{l}\text { Relaciones } \\
\text { en colegio }\end{array}$ & Enfermedad Suma \\
\hline Preoperación & 38,25 & 38 & 41,75 & 41,5 & 36,25 & 31,75 & 32,17 & 73,28 \\
Posoperación 6 meses & 15 & 15 & 16,5 & 16,5 & 17 & 15,5 & 12,83 & 55,48 \\
Posoperación 1 año & 33,75 & $41,5^{*}$ & $46^{*}$ & 43,5 & $444^{*}$ & 39,5 * & $41,5^{*}$ & $81^{*}$ \\
\hline
\end{tabular}

*Estadísticamente significativo. 
Tabla 4.

\begin{tabular}{|llllllllll|}
\hline & $\begin{array}{c}\text { Bienestar } \\
\text { físico }\end{array}$ & $\begin{array}{l}\text { Bienestar } \\
\text { emocional }\end{array}$ & Autoestima & Familia & Amigos & Colegio & Otros & Hospital & Suma \\
\hline Preoperación & 13,75 & 16,25 & 24,75 & 21 & 21,5 & 13,25 & 16,68 & 21,5 & 78,61 \\
Posoperación 6 meses & 21,75 & 16,5 & 22,5 & 23,25 & 21,25 & 22 & 44,18 & 23,3 & 78,41 \\
Posoperación 1 año & $22,25^{*}$ & $18,75^{*}$ & 26,5 & $25^{*}$ & $24,5^{*}$ & $21,25^{*}$ & $21,64^{*}$ & 21,17 & $82,6^{*}$ \\
\hline
\end{tabular}

médica de manera global en un individuo. A través de ésta hemos podido determinar en nuestra serie que en el grupo de niños menores de 7 años ha presentado cambios positivos en su calidad de vida, aun cuando la aplicación de un instrumento como el KINDL, se transforma en un desafío y quizás una desventaja en este tipo de pacientes y se puede ver influenciado por la apreciación de los padres durante el llenado de la encuesta.

Los participantes de la categoría entre 7 y 17 años presentaron resultados interesantes, tras el seguimiento realizado, se puede apreciar que tras la operación el porcentaje de quienes declaran haberse sentido enfermos aumenta, ello se puede deber a que posterior a la intervención se toma conciencia de la enfermedad, junto con las complejidades del proceso de recuperación y adaptación. De la misma forma, el $20 \%$ de los pacientes declararon sentir dolor Casi siempre, tanto a los 6 meses como en los resultados de un año posoperación, porcentaje que puede justificarse por los dolores y malestares propios del proceso de recuperación posintervención, que obviamente previo a la cirugía no se presentaban y que producen un impacto en la calidad de vida.

En general, la calidad de vida disminuye a los 6 meses posimplante coclear en todos los grupos estudiados, sin embargo al año de seguimiento se evidencian ya cambios positivos en la mayoría de las subescalas del instrumento y en la percepción global de ésta. Por otro lado, durante el seguimiento se puede apreciar que en general los adolescentes se han sentido orgullosos de sí mismos, ya que durante los 3 momentos evaluados, el $90 \%$ de las respuestas fluctúa entre Algunas veces, Casi siempre o Siempre. Lo que al cotejarlo con la mirada de los padres, demuestra que éstos también poseen la misma valoración de sus hijos sin existir cambios significativos posterior al implante coclear.
Cuando se consulta por las relaciones sociales, amigos y familia, se observa una mejoría importante posterior a la intervención, con mejor aceptación de la enfermedad, mejoría en la interacción con sus pares y una conducta en el hogar claramente favorable según la valoración parental. En temas educativos los jóvenes implantados demuestran una mayor preocupación por sus actividades escolares y por la preocupación por su futuro, lo que se puede interpretar como el aumento de las expectativas por alcanzar un nivel educacional mayor ante la superación de la barrera auditiva.

Una de las categorías de mayor relevancia que hemos abordado en el estudio, ha sido el acercamiento al ámbito emocional de los implantados y su entorno familiar. Previo a la operación la enfermedad se constituye como un motivo de tristeza, disminuyendo sus niveles porcentuales durante el proceso posoperatorio, los resultados indican que $50 \%$ de los participantes declaró en la encuesta a los 6 meses de la operación, Casi nunca haberse sentido triste por la enfermedad, y tras el año de seguimiento ello ocurre en $60 \%$ de los casos; disminución que se explica por los mismos procesos de recuperación de los pacientes, que si bien, en algunos casos resultan ser problemáticos o molestos en una dimensión de dolor, les permite proyectar una mejoría.

En relación a los núcleos familiares y entornos de los pacientes, la valoración de un mejoramiento en la calidad de vida de los pacientes es positiva, resaltando aquellas respuestas que apuntan a una mayor aceptación de su enfermedad, demostración de mayor energía y preocupación por su educación, aun cuando los pacientes se embarcan en nuevos procesos de recuperación, como es el proceso de rehabilitación auditiva; la importancia de los equipos encargados en los procesos de implantes cocleares de los centros de salud, ya sean en las etapas 
previas a la intervención, como en las evaluaciones psicológicas de los niños y grupos familiares, son parte vital para superar los desafíos posteriores a la intervención quirúrgica, compromiso que no solo toma el equipo médico, sino que también las redes de apoyo de los niños, y ellos mismos.

Revisando la literatura, observamos como en distintas publicaciones se mencionan mejorías en la calidad de vida de pacientes en los que se puede esperar un menor resultado auditivo del implante, como son los pacientes adultos. Por otra parte, varios estudios demuestran una relación pequeña aunque estadísticamente significativa entre los resultados de las pruebas audiológicas de los pacientes y los resultados de los test de calidad de vida ${ }^{13-14}$, mientras que otros autores no logran establecer esta relación ${ }^{15}$. Todas estas diferencias que surgen en la literatura internacional nos demuestran que la evaluación audiológica de los pacientes implantados no basta para conocer los beneficios que el implante coclear puede llegar a entregarles, y es por esto, que existe necesidad no solo el uso de cuestionarios de calidad de vida para

\section{BIBLIOGRAFÍA}

1. Hinderink JB, Krabbe PfM, Van den Broek P. Development and application of a health-related quality-of-life instrument for adults with cochlear implants: The Nijmegen Cochlear Implant Questionnaire. Otolaryngol Head Neck Surg 2000; 123: 756-65.

2. Palmer CS, Niparko JK, Wyatt JR, Rothman M, DE Lissovor G. A prospective study of the cost utility of the multichannel cochlear implant. Arch Otolaryngol Head Neck Surg 1999; 125: 1221-8.

3. Beadle EAR, Shores A, Wood EJ. Parental perceptions of the impact upon the family of cochlear implantation in children. Ann Otol Rhinol Laryngol 2000; 109: 111-4.

4. Rahal M, Mena P, Muñoz D, Mansilla F, Cardemil F. Resultados del Programa Nacional de Implantes Cocleares: Servicio de Otorrinolaringología del Hospital Barros Luco Trudeau. Rev Otorrinolaringol Cir Cabeza Cuello 2013; 73: 231-7.

5. Meinzen-Derr J, Lim LH, Choo Di, Buyniski S, WILEY S. Pediatric hearing impairment caregiver la correcta valoración de estos pacientes, sino que otros métodos como entrevistas realizadas por especialistas en la temática sociológica y que podrían entregar apreciaciones cualitativas y el como ellos comprenden el proceso, así como las falencias del sistema y la experiencia de quienes se someten a esta intervención.

\section{CONCLUSIÓN}

Tras el seguimiento realizado a los niños y jóvenes diagnosticados con hipoacusia e implantados en nuestro centro, su entorno social, familiar, educativo y los propios efectos de la enfermedad se puede apreciar que en general éstos han reaccionado de buena manera tras la operación, pudiendo llevar una "vida normal" con todo lo que ello implica, es decir relaciones familiares, con otros niños/as, sus estudios y el sentirse física y anímicamente bien. Por lo tanto, la calidad de vida tanto propia como de su entorno familiar ha presenciado una mejoría con el implante coclear.

experience: impact of duration of hearing loss on parental stress. Int J Pediatr Otorhinolaryngol 2008; 72: 1693-703.

6. Monteiro E, Shipp D, Chen J, Nedzelski J, Lin V. Cochlear Implantation: A Personal and Societal Economic Perspective Examining the Effects of Cochlear Implantation on Personal Income. $J$ Otolaryngol Head Neck Surg 2012; 41: 43-8.

7. Faber CE, Grontved AM. Cochlear implantation and change in quality of life. Acta Otolaryngol 2000; 543: 151-3.

8. Robinson K, Gatehouse S, Browning GG. Measuring patient Benefit from otorhinolaryngological surgery and therapy. Ann Otol Rhinol Laryngol 1996; 105: 415-22.

9. Kelsay DMR, Tyler RS. Advantages and disadvantages expected and realized by pediatric cochlear implant recipients as reported by their parents. Am J Otol 1996; 17: 866-73.

10. A. Castro, L. Lassaletta, M. Bastarrica, C. Alfonso, M. P. Prim, M. J. de Sarriá, J. Gavilán. Calidad de vida en pacientes con implante coclear. Acta Otorrinolaringol Esp 2005; 55: 192-97. 
11. Erhart M, Ellert U, Bärbel M, Ulrike R. Measuring adolescents' HRQoL via self reports and parent proxy reports: an evaluation of the psychometric properties of both versions of the KINDL-R instrument. Health and Quality of Life Outcomes 2009; 7: 77.

12. L. Rajmila, V. Serra-Suttona, J.A. Fernandez-Lopez, S. Berraa, M. Aymericha, A. Ciezac, M. Ferrerd, M. Bullingere y U. Ravens-Siebererf. Versión española del cuestionario alemán de calidad de vida relacionada con la salud en población infantil y de adolescentes: el Kindl. An Pediatr (Barc) 2004; 60: 514-21.
13. Francis HW, Chee N, Yeagle J, Cheng A, Niparko JK. Impact of cochlear implants on the functional health status of older adults. Laryngoscope 2002; 112: 1482-8.

14. Waltzman SB, Cohen NL, Shapiro WH. The benefits of cochlear implantation in the geriatric population. Otolaryngol Head Neck Surg 1993; 108: 329-33.

15. Maillet CJ, Tyler RS, Jordan HN. Change in the quality of life of adult cochlear implant patients. Ann Otol Rhinol Laryngol Supp/ 1995; 165: 3148.

Dirrección: Carlos Morales Aguirre 